\title{
Soluções Eletrônicas para Cálculos de Velocidade em Acidentes de Trânsito
}

Electronic Solutions for Velocity Evaluation in Traffic Accidents

\author{
O. Negrini Neto \\ Instituto de Criminalística do Estado de São Paulo, \\ Rua Moncorvo Filho 410, Butantã, SP, Brasil \\ Academia de Polícia Civil de São Paulo \\ Praça Reynaldo Porchat, 219 \\ Cidade Universitária, 0550\%-060, SP, Brasil
}

Recebido em 7 de março, 2002. Aceito em 11 de abril, 2002.

\begin{abstract}
As atribuições próprias do cargo de Perito Criminal, profissional responsável pelas perícias técnicas na área penal, dentre as quais as relativas a acidentes de trânsito, exigem muitas vezes conhecimentos específicos que não são ensinados em qualquer curso superior. Tais conhecimentos são fornecidos através dos cursos de formação específica, obrigatória para todos os recém ingressos na carreira, através de concurso público, bem como nos cursos de especialização, exigidos como pré-requisitos para as promoções. Destacam-se, dentre esses conhecimentos necessários, aqueles que são próprios da Física Forense. No presente artigo, apresentamos um método por nós desenvolvido e que vem sendo ensinado nestes cursos e utilizado com sucesso nos casos reais para a avaliação das velocidades de veículos colidentes. Introduzimos um tratamento eletrônico pelo uso de planilhas de cálculos que possibilitam o estudo de variações de grandezas duvidosas e de um software que permite uma rápida análise do acidente, reconstituindo-o esquematicamente e possibilitando, simultaneamente, a avaliação das velocidades, através da aplicação direta da conservação da quantidade de movimento.
\end{abstract}

The features of the job of a technical witness, who is the responsible by technical analysis associated to crime investigation, such as traffic accidents, often require specifics knowledge that are not taught in any graduation course. Such knowledge is obtained after the access to the career through professional or specialization courses, these last required to reach higher positions. Among them, there are those relied to Forensic Physics. In this issue we present a method that we have developed and that have been taught in those courses and applied successfully in real cases for the evaluation of the velocities of colliding vehicles. We have introduced an electronic treatment to perform calculations that permit studies of variations of nom-exacts parameters, besides of a fast analysis of the accident, its schematic reconstruction and the evaluation of velocities by direct application of linear momentum conservation.

\section{Introdução: a Física Forense e os acidentes de trânsito}

Nos cursos de formação técnico-profissional para Perito Criminal, a investigação técnica dos acidentes de trânsito ocupa um lugar de destaque, como parte do programa de Física Forense em cursos na Academia de Polícia Civil de São Paulo. Denominamos Física Forense a parte da Física destinada à observação, análise e interpretação dos fenômenos físicos - naturais - de interesse judiciário. Dentre estes se destacam os relativos aos acidentes de trânsito. Tais fenômenos podem ser vistos como parte da dinâmica dos corpos rígidos, sob certas condições, e assim têm sido tratados por especialistas na área [1].

Em muitos casos, não existem elementos técnicos suficientes para uma reconstrução cientificamente perfeita de um acidente de trânsito. Isso se deve, principalmente, ao fato de que a coleta de dados nos locais de ocorrências da espécie depende de vários fatores, como o tempo disponível, as condições do local, visibilidade, equipamentos, etc., nem sempre favoráveis. Em resumo, trabalha-se com o que é possível, não com o que é ideal. Assim mesmo, as colisões ocorridas em cruzamentos - perpendiculares ou não - podem ser satisfatoriamente resolvidas com aplicações direta de princípios elementares de Dinâmica, destacando-se o Princípio da Conservação da Quantidade de Movimento 
- PCQM. Sob certas condições, que normalmente são seguidas por tais tipos de acidentes, é possível calcular, com boa aproximação, as velocidades dos veículos colidentes. Esta avaliação é de extrema importância sob o ponto de vista judiciário, de vez que existe um certo preconceito de que a velocidade, ou melhor, seu excesso, é sempre a causa destas ocorrências. Esta idéia é conhecida nos meios técnicos forenses como a "hipótese do velocismo". Na realidade, muitos acidentes ocorrem por causas outras, que não a velocidade. Pode-se citar, por exemplo, o desrespeito à sinalização, a inadequada distância de segurança, a interceptação de trajetória ou ainda problemas técnicos do veículo e da via como elementos importantes nas análises de causas de um acidente. Mas não se pode negar que, até mesmo para uma comprovação de que a velocidade desenvolvida nada teve a ver com o acidente, são importantes as aproximações físicas.

O corpo discente do curso de formação técnicoprofissional é composto por recém-ingressos na carreira, após concurso público de provas e títulos, para o qual é exigido diploma de nível superior, sem especificações de áreas. Isso traz uma dificuldade adicional: como ensinar princípios físicos - que geralmente não são muito discutidos nos cursos básicos - para uma platéia tão heterogênea? A solução que encontramos, e que tem produzido bons resultados, é tornar o curso o mais prático possível, ao mesmo tempo em que os conceitos vão sendo discutidos com a profundidade adequada à profissão. Para achar este grau de profundidade, devemos conhecer as atribuições básicas da carreira: o perito criminal será o responsável pela produção da prova material científica do fato delituoso. No caso presente, sua responsabilidade implica em executar o levantamento do local do acidente, observar os vestígios encontrados, interpretá-los cientificamente e relatar suas conclusões através de seu laudo pericial. Esse relatório deve ser claro, facilmente entendido pelos profissionais da área jurídica e consistente. É claro que, quanto maior o grau de conhecimento do perito, maior será esta clareza.

\section{Programa básico do curso}

O programa do curso cobre as necessidades básicas de um curso de formação. Ao longo da carreira do perito, são oferecidos também cursos de especialização na área. Estes tratam de assuntos mais aprofundados e de discussões sobre temas em Física dos Acidentes de Trânsito.

No curso são apresentados os tópicos: Conceitos de tráfego e de trânsito, acidente de trânsito e crimes de trânsito; tipos de acidentes, unidades de tráfego. A legislação de trânsito; estabelecimento das regras de circulação; ponto de partida para análise de acidentes; as regras estabelecidas para o condutor, o veículo e a via. Causas determinantes; estudos da cadeia de pontos que se sucedem em um acidente; origem; importância do levantamento do local; as causas relacionadas ao homem; as causas relacionadas à máquina (veículo); as causas relacionadas ao meio. Velocidade; origem da metodologia da investigação; histórico dos sistemas utilizados; conceitos e nomenclaturas; processo analítico para determinar velocidades, distâncias, tempos, raios de curva e constantes de atrito; fórmulas e cálculos atuais para determinação da velocidade em veículos de passeio, utilitários, caminhões, carretas e outros. Investigação de acidente de tráfego; exame do local; conceitos e nomenclaturas; tipos e classificação das vias; delimitação da área do acidente; sistemática da investigação; levantamento topográfico e fotográfico do local; identificação e classificação das marcas pneumáticas; sítio de colisão; orientação das avarias dos veículos; identificação do veículo evasor; determinação da velocidade; dinâmica do acidente; conclusão. Exame dos veículos em geral; sistema de direção; sistema de freios, seus tipos, peculiaridades e funcionamento; sistema de segurança. Elementos de um laudo pericial; formas de apresentação, técnicas a serem adotadas, estudo dos tópicos principais que devam constar.

\section{Princípios físicos}

O princípio utilizado é o da conservação da quantidade de movimento. Admitido como boa aproximação nos meios forenses, ele permite que se avaliem as velocidades de impacto dos veículos nos acidentes, tratados como sistemas fechados. Não são feitas aproximações com base na conservação do momento angular nos tipos de acidente tratados no software; este assunto é tratado nos cursos de especialização.

Princípio da conservação da quantidade de movimento (PCQM)

O software usa o princípio de conservação da quantidade de movimento, considerando os veículos como pontos materiais: se duas partículas A e B, de massas $\mathrm{m}_{A}$ e $\mathrm{m}_{B}$ seguem determinadas trajetórias de tal sorte que, num determinado instante do tempo estarão no mesmo ponto do espaço, elas irão colidir, mas de forma tal que a quantidade de movimento total do sistema composto pelas duas partículas se mantém constante, ou seja:

$$
\sum_{i=A, B} \vec{P}_{i}=\text { const } \Rightarrow \vec{P}_{A}+\vec{P}_{B}=\vec{P}_{A}^{\prime}+\vec{P}_{B}^{\prime}
$$

(antes da colisão = após a colisão) ou :

$$
m_{A} \vec{v}_{A}+m_{B} \vec{v}_{B}=m_{A} \vec{u}_{A}+m_{B} \vec{u}_{B}
$$

onde $\vec{v}$ são as velocidades antes da colisão e $\vec{u}$ as velocidades após colidirem. 
Basicamente, é este o conceito de que precisamos para introduzir a física dos acidentes de trânsito.

Antes de estudarmos acidentes reais (v. Tabela 1), vamos utilizar um modelo de acidente para o estudo completo dos conceitos introduzidos. Consideremos uma colisão perpendicular entre os veículos 1 e 2 :

Vamos admitir que os veículos têm massas $m_{1}$ e $m_{2}$, entraram no acidente em ângulos $a_{1}$ e $a_{2}$ e que, após o acidente, adquiriram velocidades $u_{1} e u_{2}$ nas direções dadas pelos ângulos $b_{1}$ e $b_{2}$, respectivamente. Estes dados são supostos conhecidos, isto é, foram medidos ou obtidos no local do acidente pelo Perito. Em particular, as velocidades finais $u$ podem ser calculadas pelo arrastamento de pneus dos veículos após a colisão.

\section{Solução analítica da equação}

As equações (1) acima podem ser resolvidas como um sistema de duas equações a duas incógnitas. Após um simples exercício algébrico, obtemos:

$$
\begin{aligned}
& v_{1}=\frac{u_{1} \operatorname{sen}\left(a_{2}-b_{1}\right)+\frac{m_{2}}{m_{1}} u_{2} \operatorname{sen}\left(a_{2}-b_{2}\right)}{\operatorname{sen}\left(a_{2}-a_{1}\right)} \\
& v_{2}=\frac{\frac{m_{1}}{m_{2}} u_{1} \operatorname{sen}\left(b_{1}-a_{1}\right)+u_{2} \operatorname{sen}\left(b_{2}-a_{1}\right)}{\operatorname{sen}\left(a_{2}-a_{1}\right)}
\end{aligned}
$$

De posse dos valores das massas, das velocidades finais e dos ângulos, resolvemos o acidente por completo, encontrando os valores das velocidades iniciais dos veículos.

A grande vantagem da utilização do software é que ele permite simulações, isto é, havendo parâmetros com medida incerta, ou mesmo desconhecidos, através do estudo físico com uso do software é possível estabelecer-se uma faixa de valores que tornam o acidente fisicamente plausível. Isto não será exposto aqui, por não ser objetivo deste trabalho. A Tabela 1 exibe uma planilha modelo, na qual a incerteza reside na massa do veículo 1 (muitas vezes não existe informação sobre a carga transportada por um veículo, por exemplo). As soluções são fornecidas $\mathrm{em} \mathrm{m} / \mathrm{s}$ e $\mathrm{km} / \mathrm{h}$ e estão associadas ao valor da massa incerta. A última coluna mostra o valor da energia dissipada no acidente, admitida como decorrente das deformações dos veículos, que pode ser usada como parâmetro de controle.

\begin{tabular}{|c|c|c|c|c|c|c|c|c|c|c|c|c|c|c|c|}
\hline $\mathrm{m} 1$ & $\mathrm{~m} 2$ & $\mathrm{a} 1$ & $\mathrm{a} 2$ & b1 & $\mathrm{b} 2$ & $\mathrm{x} 1$ & $\mathrm{x} 2$ & atr & $\mathrm{u} 1$ & $\mathrm{u} 2$ & $\mathrm{v} 1$ & $\mathrm{v} 2$ & $\mathrm{v} 1$ & $\mathrm{v} 2$ & $\mathrm{E} \%$ \\
\hline 1900 & 1200 & 0,00 & 90,00 & 30,00 & 45,00 & 8,00 & 12,00 & 0,80 & 11,31 & 13,86 & 15,98 & 18,75 & 57,53 & 67,49 & 0,48 \\
\hline 1950 & 1200 & 0 & 00 & 30,00 &, 00 & 8,00 & 12,00 & 0,80 & 11,31 & 13,86 & 5,82 & 18,98 & 56,96 & 68,34 & 0,48 \\
\hline 2000 & 1200 & 0,00 & 90,00 & 30,00 & 45,00 & 8,00 & 12,00 & 0,80 & 11,31 & 13,86 & 15,67 & 19,22 & 56,42 & 69,18 & 0,48 \\
\hline 2050 & 1200 & $0, \mathrm{C}$ & 0 & 00 & 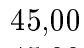 & 0 & 12 , & 0 & 11 , & 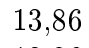 & & 5 & 55,90 & 70,03 &, 48 \\
\hline 2100 & 1200 & 0,00 & 90,00 & 30,00 & 45,00 & 8,00 & 12,00 & 0,80 & 11,31 & 13,86 & 15,39 & 19,69 & 55,41 & 70,88 & 0,48 \\
\hline 2150 & 1200 & 0,00 & 90,00 & 30,00 & 45,00 & 8,00 & 12,00 & 0,80 & 11,31 & 13,86 & 15,26 & 19,92 & 54 & 71,73 & 0,48 \\
\hline 2200 & 1200 & 0,00 & 90,00 & 30,00 & 45,00 & 8,00 & 12,00 & 0,80 & 11,31 & 13,86 & 15,14 & 20,16 & 54,49 & 72,58 & 0,48 \\
\hline 2250 & 1200 & 0,00 & 90,00 & 30,00 & 45,00 & 8,00 & 12,00 & 0,80 & 11,31 & 13,86 & 15,02 & 20,40 & 54,07 & 73,42 & 0,49 \\
\hline 2300 & 1200 & 0,00 & 90,00 & 30,00 & 45,00 & 8,00 & 12,00 & 0,80 & 11,31 & 13,86 & 14,90 & 20,63 & 53,66 & 74,27 & 0,49 \\
\hline
\end{tabular}

\section{PLANILHA DE CÁLCULOS}

Tabela 1. Exemplo de análise de acidente de trânsito (tabela abaixo) com base na planilha de cálculos. Obtidas as velocidades, é possível verificar se o acidente seria ou não evitável, bem como determinar a velocidade crítica (v'), acima da qual não seria

\begin{tabular}{|c|c|c|c|c|c|c|c|c|c|c|c|c|c|c|}
\hline $\mathrm{v} 1$ & $\mathrm{v} 2$ & $\mathrm{v} 1(\mathrm{k})$ & $\mathrm{v} 2(\mathrm{k})$ & TR & D1 & $\mathrm{T} 1$ & v'1 & $\mathrm{V}^{\prime} 1(\mathrm{~K})$ & D2 & T2 & $\mathrm{V}^{\prime} 2$ & $\mathrm{~V}^{\prime} 2(\mathrm{k})$ & D2min & D1min \\
\hline 15,98 & 18,75 & 57,53 & 67,49 & 1,50 & 30,10 & 1,85 & 12,94 & 46,59 & 28,12 & 1,15 & 12,31 & 44,31 & 34,73 & 18,33 \\
\hline 15,82 & 18,98 & 56,96 & 68,34 & 1,50 & 30,75 & 1,90 & 13,15 & 47,33 & 28,47 & 1,10 & 12,42 & 44,72 & 36,12 & 17,36 \\
\hline 15,67 & 19,22 & 56,42 & 69,18 & 1,50 & 31,40 & 1,95 & 13,35 & 48,07 & 28,83 & 1,05 & 12,54 & 45,13 & 37,52 & 16,42 \\
\hline 15,53 & 19,45 & 55,90 & 70,03 & 1,50 & 32,05 & 2,00 & 13,55 & 48,80 & 29,18 & 1,00 & 12,65 & 45,54 & 38,92 & 15,52 \\
\hline 15,39 & 19,69 & 55,41 & 70,88 & 1,50 & 32,70 & 2,05 & 13,76 & 49,52 & 29,53 & 0,95 & 12,76 & 45,95 & 40,33 & 14,65 \\
\hline 15,26 & 19,92 & 54,94 & 71,73 & 1,50 & 33,36 & 2,09 & 13,96 & 50,24 & 29,89 & 0,91 & 12,88 & 46,35 & 41,74 & 13,81 \\
\hline 15,14 & 20,16 & 54,49 & 72,58 & 1,50 & 34,01 & 2,14 & 14,16 & 50,96 & 30,24 & 0,86 & 12,99 & 46,76 & 43,16 & 13,01 \\
\hline 15,02 & 20,40 & 54,07 & 73,42 & 1,50 & 34,67 & 2,19 & 14,35 & 51,68 & 30,59 & 0,81 & 13,10 & 47,16 & 44,58 & 12,23 \\
\hline 14,90 & 20,63 & 53,66 & 74,27 & 1,50 & 35,34 & 2,23 & 14,55 & 52,39 & 30,95 & 0,77 & 13,21 & 47,56 & 46,02 & 11,47 \\
\hline
\end{tabular}
mais possível evitá-lo, e qual dos veículos teria sido o causador do evento, à luz da legislação.

Onde "TR" é o tempo de reação; "D" a distância do ponto de percepção ao da colisão e "v" a velocidade crítica para o acidente (acima da qual o acidente é inevitável. 


\section{Planilhas, Bancos de Dados e Simulação}

O software propõe prestar-se ao estudo das colisões de dois veículos nos casos de acidentes de trânsito calculando as velocidades desenvolvidas por veículos no momento do impacto, baseado no princípio da conservação da quantidade de movimento. O programa foi escrito em Visual Basic 5 da Microsoft.

A premissa básica para o uso do software é que o usuário detenha os conhecimentos básicos de Física Aplicada e os princípios referentes aos levantamentos de locais fornecidos no curso de formação. Assim, o software se torna uma ferramenta adequada para o estudo sobre as causas do acidente.

\section{Procedimentos de uso}

Em formulário próprio fornecido através de menu, o usuário informa os seguintes dados:

A qualificação dos veículos:

Placas de identificação para individualização do veículo

Marca do fabricante

Modelo do fabricante

Massa:

Do veículo

Dos passageiros

Das bagagens

(A massa deve ser informada em quilos)

Condições da pista no que se refere ao tipo de pavimentação:

Asfalto

Paralelepípedo

Terra batida

Outra.

Condições da pista:

Seca

Molhada (chuva)

Estado de Conservação:

Bom

Regular

Péssimo
Compiladas as informações, o software verifica um banco de dados fornecido, indicanndo o coeficiente de atrito correspondemente às características da pista e do veículo. A seguir, o usuário, em ambiente gráfico e com auxílio do mouse e o evento "arrasta e solta", coloca os veículos-objeto nas posições relativas conforme o ângulo assumido por eles antes do impacto. Os veículos devem ser orientados em relação a um sistema de eixos cartesianos, tendo sua origem no sítio da colisão (Ponto $(0,0))$.

Ainda com auxílio do mouse, o usuário posiciona os veículos nos pontos de imobilização assumidos após o impacto, medindo os ângulos no sentido anti-horário em relação ao eixo imaginário " $\mathrm{X}$ " e a distância (em metros) do sítio de colisão.

De forma imediata, o software calcula a velocidade de cada veículo na iminência do impacto.

Mesmo durante o evento "arrastar e soltar", a cada nova configuração assumida pelo desenho, são mostrados ao usuário a distância a partir do sítio da colisão em metros, os ângulos e velocidades de impacto, facilitando a visualização do posicionamento dos veículos, bem como o estudo do desenrolar do acidente.

Se preferir, o usuário pode entrar com os dados do posicionamento dos veículos, via teclado, em formulário fornecido por menu e o programa mostrará o resultado final imediatamente.

Um banco de dados de construções geométricas de pistas de rolamento em diversos desenvolvimentos topográficos ajuda a construir o ambiente do acidente de modo a permitir uma visão real. Tomando-se quadros sucessivos do evento, é possível reconstituir as diversas etapas do acidente. O software não utiliza recursos de animação, pois ele não seria prático para demonstrações em tribunais.

Satisfeito com o resultado, o usuário terá como opção imprimir um relatório técnico como ainda salvar em arquivo, na unidade conveniente, para visualização posterior.

Trata-se de um software de operação simples e de resultado imediato e preciso.

A Fig. 1 exibe uma tela modelo do software, com os resultados fornecidos. 


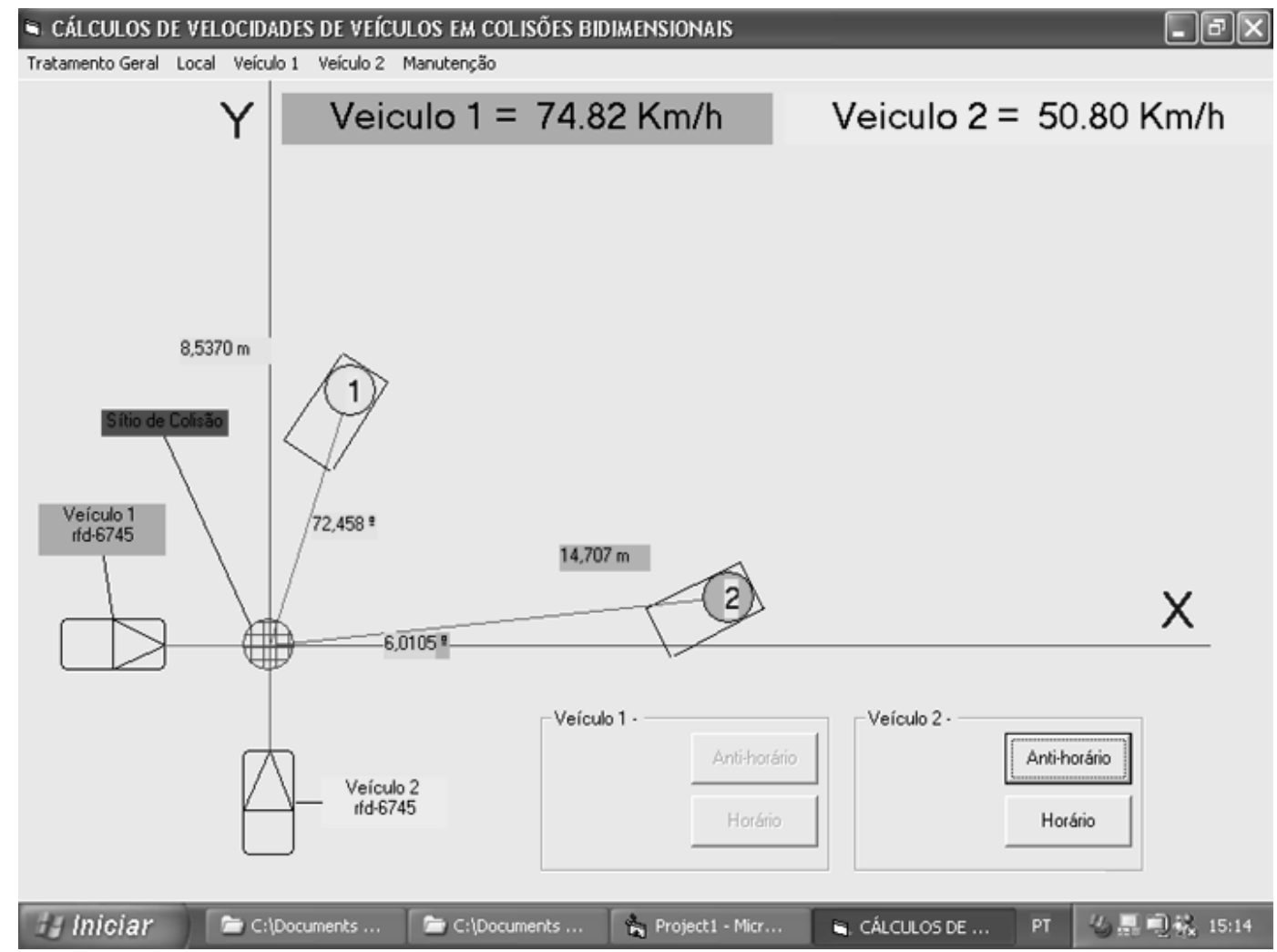

Figura 1.

\section{Agradecimentos}

O autor agradece a inestimável colaboração do Perito Criminal Onias Tavares de Aguiar, Diretor do Núcleo de Perícias de Informática do Instituto de Criminalística de São Paulo pelo auxílio na construção do software e soluções apontadas.

\section{Referências}

EUBANKS, J. Pedestrian Accident Reconstruction Lawyers \& Judges Publ., Tucson, Arizona (USA) 1994. 